\title{
Experimental Base for Introduction to Non-magnetically Fusion Engine Development
}

\author{
Baruch Boris Gutman \\ Plasma Project: USSR, SUNY, Nassau Community College, USA \\ Email address: \\ bg30002001@yahoo.com \\ To cite this article: \\ Baruch Boris Gutman. Experimental Base for Introduction to Non-magnetically Fusion Engine Development. Journal of Electrical and \\ Electronic Engineering. Vol. 5, No. 5, 2017, pp. 167-169. doi: 10.11648/j.jeee.20170505.12
}

Received: August 21, 2017; Accepted: August 28, 2017; Published: September 26, 2017

\begin{abstract}
All experimental data were received in my Plasma Lab of USSR. My US Patent [1] describes the fundamentals of non-magnetic confinement of the self-set DC current Arc Plasma Torch with vortex Arc stabilization. This Patent is based on the results of my personal experimental research of this phenomenon during a process involving pulse modulation of DC current Arc Plasma. It is my attempt to realize a non-magnetic fusion device, such as a fusion engine, utilizing a Plasma gun.
\end{abstract}

Keywords: Non-magnetically Fusion, DC Arc Plasma Torch, Pulse Modulation

\section{Introduction}

"Most fusion research is focused on "magnetic confinement" reactors that use powerful magnets to fuse hydrogen plasma into helium. One of the biggest problems with that technique is that the plasma itself spawns new magnetic fields, which play havoc with the reactions" [Steve Dent, New magnetic field theory gets us closer to nuclear fusion, Space].

Plasma guns were developed from the plasma generators utilized in space research. Plasma jets for simulating re-entry conditions of space shuttles were used to evaluate ablative materials, and later for several industrial applications like coatings, etc. utilized

Plasma generators consist of a circular orifice anode and an inner cathode enclosed in a chamber and positioned in line with the nozzle. The closed chamber has a gas inlet to stabilization of the arc by vortex. By adjusting the flow of the gas, one can force the arc column to contract in the anode-channel, creating outside of the chamber flame-like flow of high enthalpy high temperature and high velocity appear. The basic patent belongs to R. M. Gage (1957, Union carbide Inc.). Two (Plasma- dyne Inc.). Patents were received in 1960, describing high current, lowvoltage, vortex-stabilized plasma jet torches.

\section{Problem Formulation and Solution}

My US Patent [1] describes the fundamentals of nonmagnetic confinement of the self-set DC of Arc Plasma Torch with vortex Arc stabilization. This Patent is based on results of my personal research of this phenomenon during a process involving pulse modulation Plasmas in DC Arc generators.

The method requires the superposition of pulses of frequency modulation $v_{\mathrm{m}}$ on the plasma arc what is capable of support thermal nuclear synthesis in the center of the arc channel without confinement by magnetic fields.

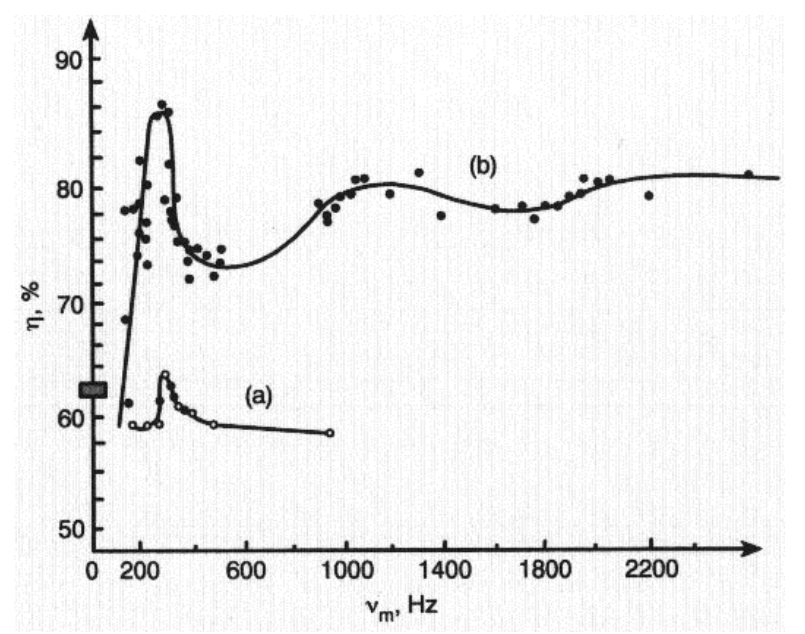

Figure 1. Comparison of torch thermal efficiency for subtraction and addition modulation. 
It could be explained by "Landau damping occurs because of the energy exchange between an electromagnetic wave with phase velocity" and electrons [2].

The intensity of conductive and convective heat exchanges in the area of resonance modulation frequencies drop down. In this case, the heat loss mostly determined by the plasma arc radiation directed from modulated DC/AC arc to a wall of the reactor channel. The rise of the heat efficiency $\eta$ at the first subtraction modulation frequency $300 \mathrm{~Hz}$ is about $25 \%$; meanwhile for "additional" pulse modulation is rise within only 3\%. Thus, "subtraction" modulation is within one order of magnitude more effective than "addition" in meaning of suppressing conductive and convective heat exchanges and consequently hindering to moving plasma fragments to walls direction.

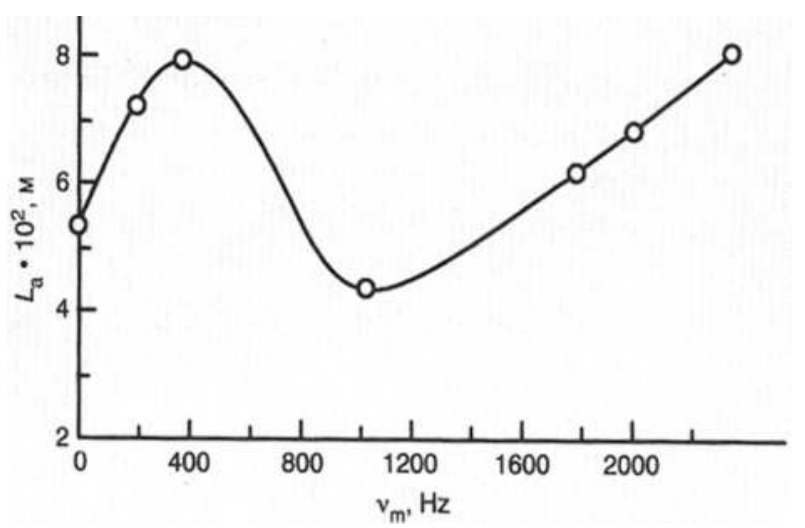

Figure 2. Length of arc La vs. modulation frequency.

The above model of plasma behavior in the arc channel supports direct measurements of length of the arc La. The length of modulated DC arc became maximal at the same resonance frequency and became equal or more than the total length of anode's arc channel. Consequently, the arc is situated in the center of arc channel. The interaction created by the pulses shock waves at certain resonance (see oscillogram) frequencies and de-energized arc may lead to a reduction of the cross component of the intrinsic vibrations of the arc. The gap existing between the arc and the anode wall tends to increase whereby the large-scale bypassing oscillations process is weakened only to contribute to the arc length growth. The process is likely moving the transition area to the anode's exit end. Given that the anode was selected to correspond as much as possible to the greatest arc length--the thermal flux going to the wall failed to intensify because of convective and conductive heat exchange occurring at the interface between the thermal and boundary layer. Whereas, the losses onto the wall were largely dependent upon the radiated heat- exchange.

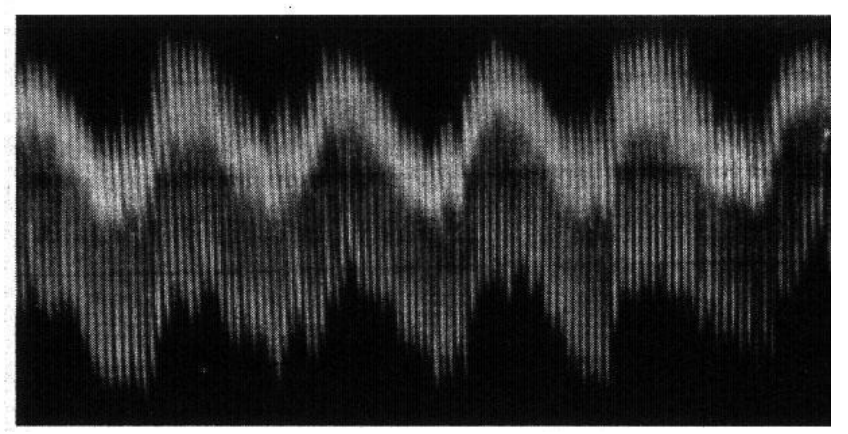

Figure 3. Current oscillogram of arc modulated by pulses.

This research was provided in a precise area of subtractive modulation resonance frequency, which is strictly sensitive to pulse, duration A: $60 \mu \mathrm{s}$ and B: $20 \mu \mathrm{s}$ ratio of current pulse amplitude $\Delta \mathrm{I}$ to an arc current, modulation frequency recurrence, high slope of the pulse (corresponding to $10^{7}-10^{8}$ $\mathrm{A} / \mathrm{s})$.

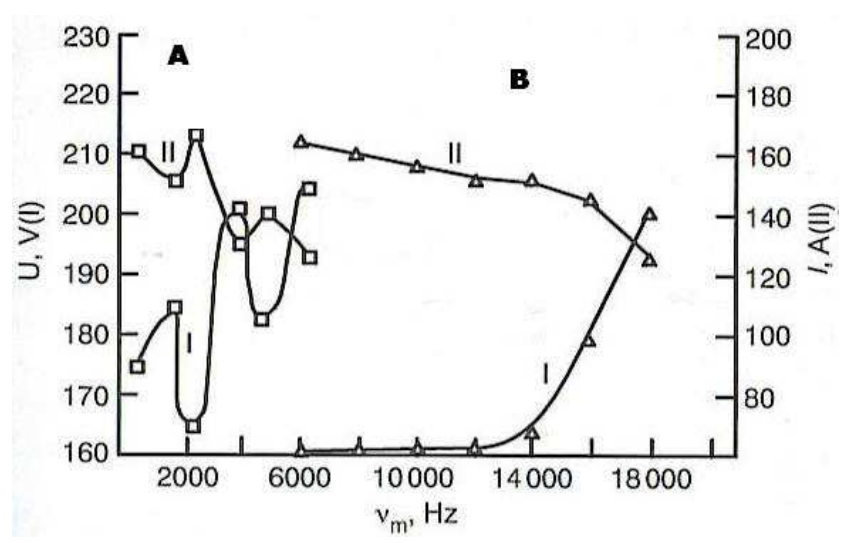

Figure 4. Dependence of arc voltage vs. pulse duration.

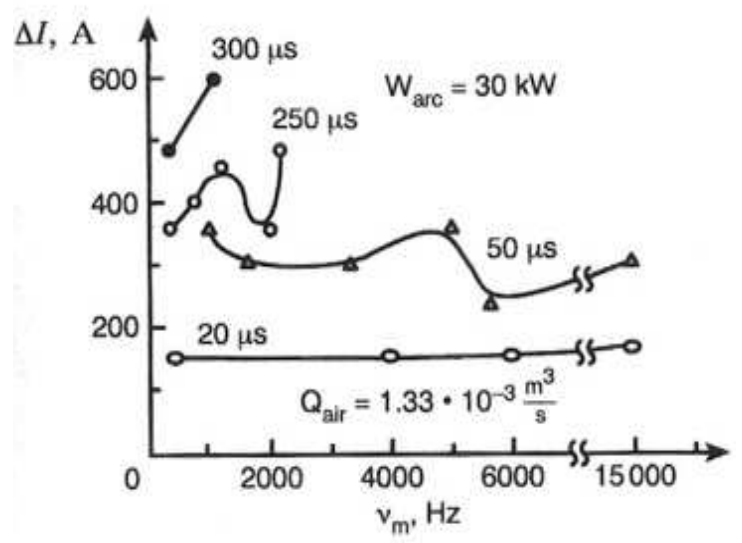

Figure 5. Dependence of pulse amplitude vs. pulse duration and frequency modulation. 


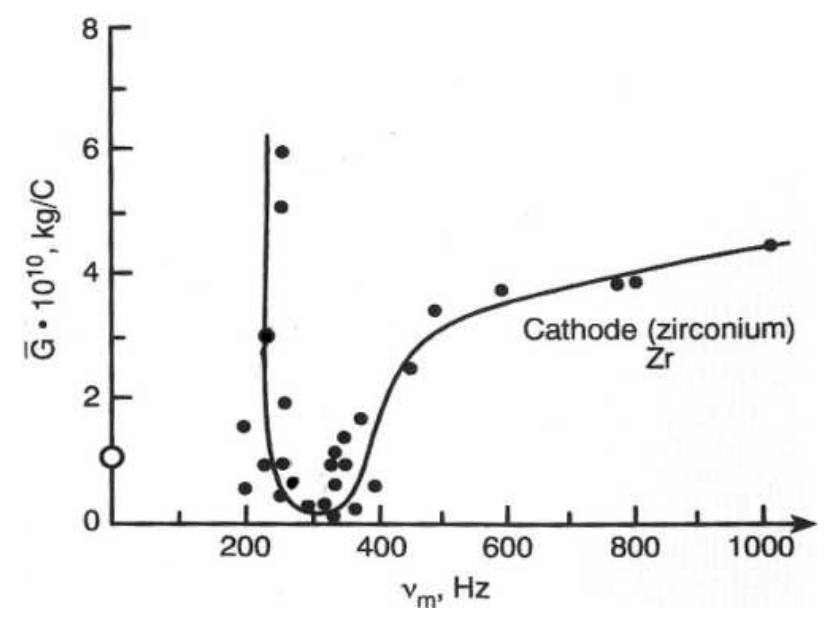

Figure 6. Dependence cathode erosion vs. frequency modulation.

Thus, modulated Plasma arc is in minimized-relatively small cross section of the center of the arc channel, corresponding to minimal cathode's erosion $\overline{\mathrm{G}}$, conductive and convective heat exchange that minimized. The arc length to be maximized, corresponding to relocate plasma reaction far from the channel's walls and consequently getting rid of significant part plasma losses of the fusion reaction to the walls.

Let us asses the physical criteria for arc swinging at $\mathrm{P}_{0}$ $=10^{5} \mathrm{~Pa}$ in accordance with an example and uncertainties from work [2]. At pulse amplitude $\Delta \mathrm{I}=0.15 \mathrm{kA}$ and pulse duration $\mathrm{t}=10 \mu \mathrm{s}$ the arc channel radius could be: $\mathrm{a}_{1}=0.93 \rho$ ${ }^{1 / 6} \Delta \mathrm{I}^{1 / 3} \mathrm{t}^{1 / 2} \sim 2.4 \cdot 10^{-3} \mathrm{~m}$. At $\Delta \mathrm{I}=1.66, \mathrm{I}$ arc $=0.25 \mathrm{kA}, \mathrm{t}=15 \mu \mathrm{s}$, $\mathrm{a}_{3} \sim 3.8 \cdot 10^{-3} \mathrm{~m}$. Pressure -to-pressure ratios at the front of a weak shock wave can be defined from the equation: $\Delta \mathrm{P}_{\mathrm{fr}}=\mathrm{Kp}$ ' $\rho \dot{a}^{2}$, where $\dot{a}=\mathrm{da} / \mathrm{dt}, \mathrm{Kp}=$ const.

We are getting $\Delta \mathrm{P}_{\mathrm{fr} 2}=\Delta \mathrm{P}_{\mathrm{fr} 1} \sim 1.1$. Thus, increasing the arc current from $150 \mathrm{~A}$ up to $250 \mathrm{~A}$ contributes to an increase in the pressure at the front, created a shock wave by pulse, is within $10 \%$ [3-9]. That could be roughly estimated by pulse current amplitude, its slop, time duration of pulse to create a fusion reaction between Deuterium and Tritium, etc.

\section{Conclusion as a Realization of Method}

Thus, the principal approach to sequence of igniting fusion in the channel of DC self-set arc could described by the following actions:
1. Set up by the pulse modulator at a resonance frequency pulse parameter, which are equal to the parameters of intrinsic arc oscillations, which is corresponding to the nonmagnetically plasma confinement from a wall thereby excluding convective and conductive heat transfer between the arc and a wall.

2. Immediately, after the above-mentioned operation, imposed, onto the modulated arc, the separate "giant" pulses + Gyrotron's radiation to heat up to fusion temperature, and compressing reaction mixture to create the fusion reaction.

3. Each fusion's explosion creates an acceleration of this space ship.

\section{References}

[1] B. Gutman, US's Patent \# 8,436,271, 2013.

[2] Landau, L. "On the vibration of the electronic plasma". J. Phys. USSR 10 (1946), 25. English translation in JETP 16, 574. Reproduced in Collected papers of L. D. Landau, edited and with an introduction by D. Haar, Pergamon Press, 1965, pp. 445-460; and in Men of Physics: L. D. Landau, Vol. 2, Pergamon Press, D. ter Haar, ed. (1965).

[3] S. N. Braginskii, Theory of the development of a spark channel, Soviet Phys, JETR (English transl.) 7, 10681074(1958).

[4] B. Gutman, Pulsed Plasma and Laser Technologies and their business aspects, Cambridge International Science Publishing, ISBN 1-898326967, 203 pages, 2000.

[5] B. Gutman-B. Goodman, US‘s Patent \# 5,900, 272, 1999.

[6] B. Gutman-B. Goodman, Israel's Patent \# 103069, 1995.

[7] B. Gutman. Pulse modulation spraying technology to produce ceramics materials for fusion and fission reactor, Poster of conference: The Nuclear Materials Conference, | Montpellier, France, 07 - 10 November 2016.

[8] B. Gutman. Introduction to non- magnetically confined fusion plasma torch-device. Poster of conference 2016, IEEE, Power modulator and high voltage conference, San Francisco, July, $5-9,2016$

[9] B. Gutman. Testing Results of Plasma Spraying Ceramics Coatings by Pulse Plasma Modulation Technology. American Journal of Nano Research and Applications. Vol. 5, No. 4, 2017, pp. 49-60. 